\title{
La Participation Citoyenne A La Gestion De La Municipalite De Guiglo En Cote d'Ivoire
}

\author{
Guiriobe Paumahoulou Jean-Arsene \\ Université Alassane Ouattara de Bouaké \\ Département d'Anthropologie et de Sociologie
}

doi: 10.19044/esj.2016.v12n11p398 URL:http://dx.doi.org/10.19044/esj.2016.v12n11p398

\begin{abstract}
These writings evaluate citizen participation in the management of municipality of Guiglo in Côte d'Ivoire. To do this, we interviewed, using individual semi-structured interviews, resource persons in this locality. From these interviews, it appears that there is no form of citizen participation in the management of that local authority, outside the municipal elections. However, local elected officials use various strategies such as the presence of representatives of populations at regular sessions of municipal councils to pretend they associate third parties in the management of their city. This lack of citizen participation is, ever so slightly, causing the mismatch between insiders' local development projects provided by that municipality and the real aspirations of the beneficiaries.
\end{abstract}

Keywords: Citizen participation, representative populations, management of local authorities, councils, municipalities

\section{Resume}

Ces écrits évaluent la participation citoyenne à la gestion de la municipalité de Guiglo en Côte d'Ivoire. Pour ce faire, l’on a interrogé, à l'aide d'entretiens semi-directifs individuels, des personnes ressources dans cette localité. Il ressort de ces entretiens qu'il n'existe aucune forme de participation citoyenne à la gestion de cette collectivité territoriale, en dehors des élections municipales. Les autorités locales utilisent cependant diverses stratégies telle que la présence des représentants des populations aux différentes sessions ordinaires des conseils municipaux afin de donner l'impression qu'elles associent des personnes tierces à la gestion de la cité, alors que dans le fond il n'en est rien. Cette inexistence de participation citoyenne est, un tant soit peu, à l'origine de l'inadéquation entre les projets de développement local initiés et réalisés par cette instance décentralisée et les aspirations réelles des populations bénéficiaires. 
Mots clés : Participation citoyenne, représentants de population, gestion, collectivités locales, conseillers municipaux, communes, municipalités

\section{Introduction}

La participation citoyenne à la gestion des collectivités locales exprime la mesure selon laquelle les autorités locales prennent en compte les avis et les préoccupations de la société civile en dehors des périodes électorales (CGLU Afrique et Cities Alliance, 2013 :14). Elle inclut divers processus de consultation dont les mécanismes doivent être formalisés pour qu'ils soient efficaces: la possibilité pour les citoyens de présenter des pétitions, l'organisation de référendum d'initiative citoyenne, la pratique du budget participatif, l'expression des avis des citoyens à travers les enquêtes de satisfaction sur les différents sujets qui les intéressent, etc. (CGLU Afrique et Cities Alliance, op. cit:15). Ces diverses formes de participation citoyenne à la gestion des collectivités locales doivent, en effet, être prescrites et organisées par les lois et règlements et faire l'objet de suivi dans la mise en œuvre, au niveau central comme au niveau local, pour être réellement intégrées dans les pratiques des collectivités locales, car les conditions de réalisation d'une bonne gouvernance locale dans le cadre de la décentralisation dépendent du degré d’appropriation du processus par les populations et, surtout la bonne gestion des instances décentralisées ne saurait prospérer sans cette participation (Center for Research on Local Knowledge, 2007 : 02) . Au Burkina Faso, la loi prévoit dans ce cadre, la mise en place d'un conseil villageois de développement (CVD) dans chaque village autre que le chef lieu de la commune. Les membres de ces conseils sont élus par les villageois sous la supervision des conseils des collectivités locales (NDI, 2010 :18). En Tunisie, pour promouvoir la participation citoyenne à l'action municipale, la nouvelle constitution de 2014 a mis l'accent sur deux principaux moyens. Il s'agit des instruments de droit politique formel et les mécanismes participatifs (Wiedemann et Gafsi, 2014 :11). Cependant, en Côte d'Ivoire où une politique de décentralisation est également mise en œuvre depuis plusieurs décennies, aucune forme de participation citoyenne n'est prescrite et organisée par des lois et règlements afin d'intégrer effectivement celle-ci dans les pratiques des collectivités locales. Dans ce contexte, comment les populations de la commune de Guiglo participent-elles à la gestion de leur collectivité locale?

L’objectif de cette recherche est d'évaluer la participation citoyenne à la gestion de la municipalité de Guiglo, en l'absence de cadre juridique devant réglementer ce secteur en Côte d'Ivoire. Pour atteindre cet objectif, l'on a interrogé des personnes ressources. Les résultats obtenus sont exposés à travers trois points majeurs que sont: l'inexistence d'une véritable 
participation citoyenne dans cette commune, l'élaboration de stratégies par les élus locaux afin de donner l'impression de l'implication des citoyens à leur propre développement et enfin l'impact de telles pratiques sur le processus de décentralisation en cours et les projets de développement local.

\section{Materiel et methodes}

Les municipalités ivoiriennes n'ont pas de relations directes avec l'ensemble des populations, mais des rapports indirects qui passent par l'intermédiaire des représentants des communautés de base et de la société civile locale. C'est la raison pour laquelle cette recherche a été circonscrite autour de ces derniers ainsi que des chefs de service et des élus locaux de la municipalité visitée. Un total de 30 enquêtés, sélectionné dans cette commune à l'aide de la technique d'échantillonnage par choix raisonné, a constitué l'échantillon de cette étude. Ils sont repartis comme suit: 10 représentants des communautés de base, 10 responsables de la société civile locale et 10 agents et élus locaux de la mairie de Guiglo. Par le biais d'entretiens semi-directifs individuels, nous avons abordé, avec ces personnes, des questions concernant la pratique de la participation citoyenne à la gestion de la municipalité de Guiglo, le cadre organisationnel de cette participation et les retombées d'une telle démarche. Les informations collectées nous ont permis, d'une part, de constater l'inexistence de participation citoyenne à la gestion de la municipalité, d'autre part, elles ont contribué à l'identification des stratégies mises en place par les autorités locales afin de donner l'impression qu'elles associent les populations à la gestion de leur cité. Le choix de cette commune, située à l'ouest de la Côte d'Ivoire, à plus de cinq cents kilomètres d'Abidjan, se justifie par deux raisons essentielles. La première raison est liée au fait que ladite commune, créée au cours des années 80, fait partie des anciennes collectivités locales du pays, dotée par conséquent d'une certaine expérience en la matière. La seconde se justifie par le fait qu'elle administre tant des populations urbaines que rurales, c'est-à-dire les deux catégories de citoyens constituant la population ivoirienne (urbaine et rurale).

Les données recueillies ont par la suite été analysées selon la théorie stratégique qui définit le pouvoir comme la capacité d'un acteur à structurer des processus d'échanges plus ou moins durables en sa faveur (Friedberg, 1993 :17). La participation directe ou indirecte des populations à la gestion des collectivités locales rentre dans le cadre d'une coopération entre deux acteurs clés (les élus locaux et les populations) du processus de décentralisation. Or, la coopération s’inscrit dans des jeux d'acteurs, champ de prédilection de l'analyse stratégique.

Cette théorie permet, d'une part, de mettre en exergue les rapports existant entre les élus locaux et les citoyens ; d'autre part, elle contribue à 
identifier les différentes stratégies utilisées par les élus locaux afin d’écarter les citoyens de la gestion de leur cité.

\section{Resultats de la recherche \\ Inexistence de participation citoyenne à la gestion de la commune de Guiglo}

Dans la commune de Guiglo, après les élections municipales, les populations restent totalement à l'écart de la gestion des affaires communales, comme le témoignent le secrétaire général et le chef comptable de cette municipalité : «Dans cette commune, l'on ne peut pas vraiment parler de participation des populations à la gestion des affaires communales car les élus locaux ne leur donnent pas cette occasion » (Secrétaire Général de la mairie) ; "Les populations de cette commune ne sont associées à la vie communale que lors des élections locales, passée cette période, elles sont complètement écartées de toute prise de décision »(Chef comptable). Aussi, ces populations ne sont-elles informées de rien, dans la plupart du temps, comme le soulignent les propos du président des jeunes du village de Glopaoudi dans la commune et ceux du secrétaire général de la mairie : «Les populations ne sont ni informées ni consultées à propos des sujets qui touchent à la vie ou à la gestion de leur commune car ceux qui doivent en principe le faire ne le font pas » (Président des jeunes) ; "Les représentants des différentes communautés de base que les élus locaux invitent aux sessions ordinaires du conseil municipal, qu'ils soient chefs de villages ou de quartiers ou même conseillers municipaux, sont coupés des populations qu'ils représentent parce qu'ils ne communiquent pas avec elles comme le demande la situation » (Secrétaire général). La gestion, la vie et les affaires de la commune de Guiglo est donc l'affaire d'une poignée d'individus et non de l'ensemble des citoyens, affirment si bien le chef comptable et un chef notable de Domobly, une autre localité rurale de cette commune: «Les populations ne sont aucunement associées à la gestion des collectivités locales en Côte d'Ivoire car les autorités locales choisissent elles-mêmes des individus avec lesquels elles collaborent». (Chef comptable); "Nous ne participons pas véritablement à la gestion de la commune car on ne nous associe pas vraiment à cette gestion comme il le faut, même pour les activités de développement nous concernant et cela est regrettable »(Chef notable). Ces situations font dire aux uns et aux autres que la participation des populations à la gestion de la commune est inefficace voire très faible, comme le souligne le secrétaire général de la mairie : «Le processus de participation citoyenne à la gestion des collectivités locales dans notre commune n'en est pas un à proprement parler car il n'est pas du tout efficace ». Un chef notable interrogé à Mona, un village communal, va plus loin sur cette lancée en décriant l'inexistence de la participation citoyenne à 
la gestion de la municipalité de Guiglo en ces termes : «On ne peut pas parler d'une quelconque participation des populations à la gestion de la commune de Guiglo car elle ne transparaît nulle part ici ».

Ainsi, dans le cas de cette commune, l'on ne peut-il parler de participation des populations à la gestion de leur collectivité locale car elle n'existe sous aucune forme. Cependant, les autorités municipales utilisent diverses stratégies afin de donner l'impression d'une quelconque implication de ces populations à la vie communale.

\section{Stratégies élaborées par les autorités locales afin de donner l'impression d'une participation citoyenne à la gestion de la municipalité de Guiglo.}

En Côte d'Ivoire, il n'existe pas de lois, ni de règlements organisant, dans un contexte légal, la participation des populations à la gestion des collectivités locales. Cependant, celles-ci sont conviées aux différentes sessions ordinaires des conseils municipaux car elles sont publiques, comme le témoignent si bien les propos du secrétaire général et le chef comptable de la municipalité visitée : "Les populations sont invitées à s'exprimer sur la vie de leur commune, en donnant leurs avis et leurs préoccupations sur certains faits et actes de celle-ci, lors des sessions ordinaires qui sont d'ailleurs publiques » (Secrétaire général) ; "C'est au cours des sessions ordinaires du conseil municipal organisées généralement en fin d'année que l'occasion est donnée aux populations de se prononcer sur les différents projets initiés par la commune et d'exposer leurs différents besoins » (Chef comptable). Même son de cloche au niveau des citoyens interrogés qui reconnaissent, pour certains d'entre eux, avoir participé à ces sessions, comme le disent le chef de Zouan, village de ladite commune et une présidente d'association féminine locale : «Le maire nous convoque souvent pour assister à des réunions de travail avec son équipe » (Chef de village) ; «De temps en temps, nous allons assister à des réunions à la mairie sur la convocation du maire » (Présidente d'association féminine).

En effet, dans la commune de Guiglo, c'est généralement en fin d’année ou au dernier trimestre de l'année que sont organisées les sessions ordinaires pour procéder au bilan de l’année écoulée et faire, par la même occasion, les prévisions de l'année suivante. C'est ce que révèlent les propos de certains enquêtés tels que le premier et le deuxième adjoint au maire, et l'un des responsables de la jeunesse de Goya, une localité rurale de Guiglo : «C'est généralement en fin d'année que le conseil municipal organise une session ordinaire à laquelle les populations sont conviées, afin de faire le bilan des activités de la mairie » (Premier adjoint au maire); "En fin d'année, c'est-à-dire après le 30-09 de l'année en cours, le conseil municipal tient sa session ordinaire à laquelle les populations et leurs différents représentants sont invités » (Deuxième adjoint au maire) ; «On ne 
nous invite qu'aux réunions de fin d'année organisées par le maire où il nous dit ce qu'il a fait dans la commune et ce qui reste à faire» (Responsable de jeunesse).

Les sessions ordinaires des conseils municipaux sont certes publiques mais, ceux qui y participent, en tant qu'invités et dont les frais de déplacement et autres sont pris en charge par la mairie, sont généralement les représentants des populations et les responsables de certaines ONG locales, avancent le secrétaire général et le chef comptable de la mairie à travers les propos suivants : «Avant la tenue d'une session ordinaire, le maire, qui doit la présider, invite, en prenant en charge leurs frais de déplacement et même leur nourriture, les différents chefs des communautés de base de la commune, les responsables de la société civile locale et les opérateurs économiques locaux» (Secrétaire général) ; "Le maire et son équipe font venir les responsables des diverses structures et localités se trouvant sur le territoire communal ou ayant des intérêts dans la commune afin qu'ils prennent part, en tant que représentants de ces structures et localités, à la session qui doit se tenir les jours suivants » (Chef comptable). A la suite de ces derniers, un responsable d'une ONG locale et un président de jeunes d'un village de la commune ajoutent en ces termes: "Pour les réunions $d u$ conseil municipal, le maire invite les chefs de communautés de base, de quartiers, de villages et autres représentants de la population, qui peuvent y aller s'ils sont disponibles » (Responsable d'ONG) ; "Très souvent, ce sont les chefs notables, et d'autres responsables de structures villageoises sélectionnés par le maire lui-même qui assistent aux différentes réunions à la mairie » (Président de jeunes). Aussi, les conseillers municipaux de la majorité communale jouent-ils le rôle de représentants de populations lors de ces séances et, c'est ce que mentionnent les propos du chef comptable de la mairie de Guiglo : "Les conseillers municipaux issus du même bord que le maire sont automatiquement les représentants des populations de leur zone d'habitation ou d'origine, c'est pourquoi il nomme au moins un conseiller municipal par localité ».

Les représentants des communautés de base et les responsables de la société civile locale invités aux sessions ordinaires du conseil municipal doivent consulter leur base ou structure avant d'aller participer à ces sessions, comme le précise le secrétaire général de la mairie de Guiglo : «Les représentants invités doivent d'abord informer, consulter et concerter leur base afin de recueillir leurs différents avis et préoccupations qu'ils sont censés exposer intégralement lors de la session à laquelle ils prendront part et, à leur retour, ils doivent également rendre compte, à leurs populations, de ce qui a été décidé par le conseil municipal. Même les conseillers municipaux, qui sont aussi des représentants de la population auprès de la mairie, doivent également procéder de la même manière » 
Durant les assemblées ordinaires du conseil municipal, la parole est en effet donnée à tous les participants afin d'exprimer leurs avis et préoccupations, comme le témoignent les propos du chef comptable de la mairie de Guiglo : "Tous ceux qui sont présents à la séance ou assemblée ou encore à la session peuvent en principe prendre la parole pour exprimer leurs points de vue, leurs préoccupations, leurs besoins ou même protester contre un fait ou acte qu'ils jugent injuste ». C'est ce que révèlent également les témoignages d'un conseiller municipal et d'un adjoint au maire interrogés : "La parole est donnée à tous les représentants des populations, c'est-à-dire les chefs de village, de quartiers et autres invités à la session du conseil municipal, ce qui leur permet d'exposer les différents besoins de leur village respectif» (Conseiller municipal); «Pendant les sessions ordinaires du conseil municipal, tous ceux qui désirent exprimer un point de vue ou un avis sur la gestion des affaires de la commune peuvent le faire pleinement s'ils sont présents ». (Adjoint au maire). Mais, l'un des chefs notables du village de Zouan et un responsable de la société civile locale, interrogés sur ce fait, affirment le contraire en ces termes : "Lorsqu'on nous invite à une réunion à la mairie, on ne nous permet pas de prendre la parole afin de dire réellement ce que nous voulons pour notre village ou même ce que nous pensons du travail fait par le maire » (Chef notable) ; "L'on nous associe à ces sessions en qualité de spectateurs puisqu'on ne nous donne pas vraiment la parole » (Responsable de la société civile locale).

A côté de ce premier volet de stratégies,existe un second qui concerne les courriers adressés à la mairie par les représentants des différentes communautés de base et, c'est dans ce cadre que s'inscrivent les témoignages du secrétaire général, chef comptable et de certains représentants de communauté de base interrogés : "Parfois, il est demandé aux chefs de villages et de quartiers d'adresser des courriers contenant leurs avis et préoccupations au maire » (Secrétaire général de la mairie ); "En dehors des sessions ordinaires du conseil municipal, les représentants des populations peuvent adresser des courriers au maire pour exprimer les différentes préoccupations de leur communauté » (Chef comptable) ; "On nous demande souvent d'adresser des courriers au maire pour dire ce que nous voulons » (Un Chef notable communal).

A la suite de ces faits, nous dirons que les autorités locales ou les élus locaux de Guiglo considèrent la présence de certains représentants de communautés de base et responsables de la société civile locale aux différentes réunions et sessions ordinaires du conseil municipal comme une participation citoyenne. Mais en réalité, une présence ou une participation à une réunion du conseil municipal n'est pas une participation citoyenne à proprement parler. Elle n'est qu'une stratégie pour donner une impression de la pratique effective de la participation citoyenne dans leur cité. Quel peut 
donc être l’impact de tels agissements sur le processus de décentralisation et les projets de développement local ?

\section{Impact sur le processus de décentralisation et les projets de développement local}

Le processus de décentralisation se fait pour la population et avec la population. Mais, dans la commune de Guiglo, l'on constate que cette décentralisation se fait sans les populations, comme le font remarquer le chef comptable de la mairie de Guiglo et le président des jeunes de Kati, une localité villageoise de la commune, "Les populations ne se sentent pas encore concernées par le processus de décentralisation en cours car pour elles c'est une affaire qui ne concernent que les maires et leurs adjoints » (Chef comptable de la mairie) ; "Normalement, le pouvoir, au niveau local, devait être déconcentré et partagé avec la population, ce qui n'est encore le cas ici où nous sommes écartés du processus. Dans ce cas précis, nous ne comprenons pas le sens véritable de cette politique de décentralisation mise en œuvre » (Président des jeunes de Kati). Le secrétaire général et la présidente des femmes de Domobly vont plus loin sur cette même lancée en affirmant ceci : "Sans une participation citoyenne, un processus de décentralisation ne peut pas atteindre son objectif ultime qui est l'amélioration des conditions de vie des populations à la base, car les préoccupations réelles de celles-ci ne sont pas prises en compte. Or, c'est ce qui se passe réellement dans cette commune " (Secrétaire Général de la mairie) ; "Un processus de décentralisation dans lequel les populations ne sont pas impliquées véritablement, comme c'est le cas ici, ne peut être correctement noté » (La présidente des femmes de Domobly).

Aussi, la non implication des populations à la gestion de la commune de Guiglo provoque-t-elle une inadéquation entre les projets de développement local conçus et réalisés par ladite commune, comme le témoignent un chef notable et un responsable de la société civile locale : "Quand les populations ne sont pas impliquées dans la gestion de leur commune, comme c'est le cas ici, les maires ne peuvent pas connaître réellement leurs besoins, ce qui fait qu'ils réalisent très souvent des infrastructures qui ne cadrent pas avec les préférences de ces dernières » (Chef notable de Kati)) ; "La conséquence majeure de cette situation est l'inadéquation entre les projets de développement local réalisés et les besoins réels des populations bénéficiaires » (Responsable d'une ONG dans la commune de Guiglo).

Au regard de ce qui précède, nous retiendrons que l'inexistence de participation citoyenne à la gestion de la commune de Guiglo entraîne, d'une part, l'inefficacité du processus de décentralisation; d'autre part, elle 
occasionne une inadéquation entre les projets de développement local réalisés par la mairie et les aspirations réelles des populations bénéficiaires.

\section{Analyse et discussion}

Evaluer la pratique de la participation citoyenne dans la gestion de la municipalité de Guiglo, en l'absence de cadre juridique réglementant ce secteur en Côte d’Ivoire, est le but poursuivi au cours de cette étude.

Les résultats ont fait ressortir une inexistence de la participation des populations à la gestion de la commune de Guiglo. Ces résultats sont en effet différents de ceux obtenus en 2008 dans le cadre du DRSP en Côte d'Ivoire. En effet, en 2008, le taux de participation des populations aux actions de développement local en Côte d'Ivoire était chiffré à 25\% et il devrait atteindre 75\% en 2015 (DSRP, 2009 : 26). Ils sont cependant identiques à ceux relevés en Guinée lors d'une étude sur le renforcement de la gouvernance locale dans les zones minières du littoral. Dans ces zones il a été constaté une absence totale de contrôle citoyen sur des actions publiques locales, ce qui découle d'une inexistence de la participation citoyenne au niveau de ces actions (CADES/Guinée, 2012 : 02). La différence entre ces résultats, c'est-à-dire les résultats du DSRP et ceux de cette recherche, s’explique par le fait que pour cette étude, nous avons considéré la participation citoyenne comme un processus obligatoire ou volontaire de personnes ordinaires, agissant seules ou au sein d'une organisation, en vue d'influer sur une décision portant sur des choix significatifs qui toucherons leur communauté (Ghidouche Y. et al., 2016 : 13). Elle a été également considérée comme le fait de donner la priorité aux populations dans les projets de développement, c'est exactement leur donner les moyens d'être des acteurs sociaux plutôt que des sujets passifs afin qu'elles puissent maîtriser les activités qui affectent leur existence (Decoster, 2012 : 12). Cette conception permet d'écarter systématiquement les discours officiels des autorités locales relatifs à l'action citoyenne dans leur commune et qui masquent la réalité des faits. En effet, l'implication réelle des populations requiert de les associer à toutes les initiatives de développement (Koffi 2010 : 98). Elle est utilisée par une multitude d'acteurs (communautaires, privés et institutionnels) pour soutenir le développement des communautés, dans des initiatives diversifiées, orientées vers des perspectives de développement social, développement local, revitalisation des quartiers ou territoires appauvris (Mercier 2008 : 5). Dans cette situation, le maire et son équipe doivent saisir l'opportunité pour associer les populations à la politique municipale, notamment lors de l'élaboration du budget participatif, plan local de développement et de la gestion des structures locales. Ainsi, tous les citoyens désireux de s’impliquer dans des initiatives d’intérêt commun ou dans des projets plus ambitieux doivent être entendus et 
soutenus (Association Internationale des maires francophones 2011 : 13). Sur cette lancée, les villes tunisiennes sont-elles devenues depuis le 14 janvier 2011, le théâtre de la participation citoyenne, à travers les initiatives des collectivités locales, des citoyens et de la société civile pour prendre part à la gestion des affaires locales et aux choix qui engagent l'avenir des villes et de leurs habitants (Wiedermann et Gafsi, op. cit: 14). Ces faits avérés de participation citoyenne enregistrés dans ces villes ou endroits sont en fait loin de ceux révélés par les résultats de cette étude. En effet, dans la commune de Guiglo, la gestion de la municipalité et des projets de développement local reste l'affaire du maire et de son entourage immédiat, c'est-à-dire ses adjoints. Les populations ne sont ni informées ni consultées ni même associées aux différentes prises de décisions engageant l'existence de leur communauté. Nous constatons donc que le processus de décentralisation se déroule dans cette ville sans la population pour laquelle il a été mis en place. Et, les autorités locales essaient, à travers leurs discours et certains de leurs actes, de masquer cette réalité des faits en donnant l'impression qu'elles associent les populations à la gestion des affaires communales.

Pour atteindre cet objectif, les résultats ont révélés que les élus locaux usent de stratégies telle que la présence de certains représentants de communautés de base et responsables de la société civile locale à des réunions et sessions ordinaires du conseil municipal. Les autorités locales de Guiglo multiplient, en effet, les initiatives et les dispositifs, c'est-à-dire les stratégies pour pouvoir écarter leurs populations de la gestion des affaires locales. Elles préfèrent les inviter à assister simplement à certaines réunions du conseil municipal, au lieu de les impliquer concrètement dans les initiatives de développement local. Cette manière de faire découle d'une situation stratégique mise en place afin d'éviter la pression des populations, ce qui leur sera très bénéfique dans la conduite des affaires locales (Friedberg,op. cit.). Accepter que les populations deviennent constamment actives n'est pas chose souhaitée pour les décideurs locaux; qu'elles cherchent à s'informer, à comprendre, à donner leurs avis, à s'affirmer en qualité d'experts, qu'elles entrent dans des mécanismes de co-institution, évaluent et contrôlent les choix effectués par les élus apparaît en fait comme une véritable révolution qu'ils cherchent à éviter (Atta, 2010 : 09). Pour ce faire, les citoyens et autres organisations de la société civile font en effet l'objet de très peu d'appui et de formation à même de leur permettre de comprendre et donc de participer véritablement au processus de décentralisation et de développement local. En outre, les populations disposent de très peu de moyens de contrôle et de suivi des décisions prises (Koffi, op. cit.). Cet état des faits et son maintient sont très favorables aux élus locaux car collégialiser la gestion municipale et impliquer d'avantage la 
population dans la conduite des affaires communales ne leur paraissent pas des urgences à satisfaire car une telle implication constitue en fin de compte l'un des principaux contre-pouvoirs au niveau local (Thoenig 1996 :20). Mais, puisqu'ils veulent donner des résultats positifs en matière de participation citoyenne dans leur localité pour séduire d’éventuels bailleurs de fonds, ils invitent certains chefs de communautés de base et responsables de la société civile locale, sélectionnés par eux-mêmes afin de participer aux différentes sessions ordinaires du conseil municipal. Ces derniers sont en principe chargés de recueillir les avis et les préoccupations de ceux qu'ils sont censés représenter avant d'aller assister aux assemblées afin de les exprimer une fois présents à ces séances et à leur retour, ils doivent rendre compte, ce qui n’est pas vraiment le cas selon les résultats. En fait, dans la forme, les élus locaux s'entourent de représentants de populations et de conseillers municipaux pour donner l'impression qu'ils associent d'avantage des groupes tiers, sinon l'ensemble de la population à la gestion de leur collectivité locale. Pourtant, dans la pratique ils ne donnent véritablement la parole et n'écoutent que ceux qui sont acquis à leur cause ou qui leur sont politiquement et culturellement favorables. Une telle participation citoyenne ressemble plutôt à du bricolage (Thoenig op. cit:25). Enfin de compte, nous dirons que tout se passe en fait comme si la réflexion interne sur l'appropriation des mécanismes de la participation citoyenne était encore au stade zéro. Le pouvoir local n’est pas déconcentré et partagé avec les populations comme l'exige toute politique de décentralisation et de développement local (Akindès 2002 : 23). Et, cela engendre des effets néfastes sur le processus de décentralisation et la politique de développement local.

Les conditions de réalisation d'une bonne gouvernance locale dans le cadre de la décentralisation dépendent du degré d'appropriation du processus par les populations. Plus l'on note l'existence de contre-pouvoirs et d'obligation de reddition de comptes par les responsables locaux à ceux qui les ont mandatés, plus la gouvernance s’en trouve améliorée et le processus de décentralisation consolidé (Center for Research on Local Knowledge, op. cit.). Mais, avec une absence ou inexistence de la participation des populations, comme c'est le cas dans la commune de Guiglo, le processus de décentralisation, doté d'une certaine inefficacité comme l'ont démontré les résultats, n’est nullement consolidé et obtient une note passable, c'est-à-dire tout juste la moyenne qui est de 20/40 (CGLU et Cities Alliance, op. cit:42). En outre, cette situation engendre aussi une inadéquation entre les projets de développement local réalisés par la municipalité et les aspirations réelles des populations (Boizo, 1994 : 417). Et, cela est dû au fait que les populations ne sont pas associées au processus d'identification et de conception des projets de développement dont elles doivent bénéficier (Guiriobé, 2015 : 37). Enfin, 
il est également démontré que l'absence de tout contrôle citoyen sur les actions publiques locales dans une commune comme celle de Guiglo occasionne un manque de transparence et de redevabilité citoyenne dans la gestion des affaires publiques locales, ce qui entraîne une certaine impunité des élus et dirigeants locaux dans la pratique de malversation financière et surtout l'inefficacité de la capacité d'action en matière de développement local.

\section{Conclusion}

Notre objectif, en entreprenant cette étude, était d'évaluer la participation citoyenne à la gestion de la commune de Guiglo en Côte d'Ivoire où il n'existe aucune législation en la matière. Dans cette localité, en dehors de la participation aux élections locales, l'implication des citoyens à la gestion de leur commune n'existe sous aucune forme. Pour masquer cette anomalie, les autorités locales invitent les représentants des communautés de base et les responsables de la société civile locale aux différentes sessions ordinaires du conseil municipal qui se tiennent généralement au cours du dernier trimestre de l'année. Celles-ci sont ouvertes au grand public mais enregistrent généralement la présence des représentants des populations sélectionnés par les élus locaux eux-mêmes. Ce sont ces derniers qui sont chargés de s'exprimer au nom de la population. Mais, ils n'arrivent pas très souvent à prendre la parole lors de ces séances ou bien s'ils le font, les autorités locales ne les écoutent pas parce que leur présence n’est qu'une simple stratégie pour donner l'impression d'une quelconque participation de tierces personnes à la gestion de la cité. Une telle manière de faire engendre des effets néfastes sur la capacité d'action de la municipalité de Guiglo et les projets de développement local réalisés par cette municipalité car ils ne correspondent pas aux préférences des populations, ce qui fait qu'ils n'arrivent pas à améliorer leurs conditions de vie. Vu ces conséquences, un renforcement des capacités de ces deux catégories d'acteurs en la matière est donc nécessaire et urgent car l'implication véritable des populations à la gestion des collectivités territoriales et à leur propre développement est l'une des conditions fondamentales du succès d'un processus de décentralisation et de toute politique de développement local.

\section{References:}

Akindès F., 2002, Décentralisation, société civile et participation au développement local : des concepts à la réalité, Abidjan, PUCI, 53 p.

Association Internationale de maires francophones (AIMF), 2011, La gestion participative : guide à l'attention des collectivités territoriales, AIMF, $18 \mathrm{p}$. Atta H., 2010, Dossier de politique économique: participation des populations dans la mise en ceuvre du DRSP en Côte d'Ivoire, ACBF/ 
Institut de la Banque mondiale, Abidjan, consulté sur internet : http:// www. grpe-afrique. net/abidjan/TROMBINOSCOPE/DPE/Atta. Pdf, 33 p.

Boizo O., 1994, « Crise, gestion municipale et participation populaire ou jeu de pouvoir dans les villes », In Crise, ajustement et recomposition en Côte d'Ivoire, ORSTOM / CORDIS, Abidjan, pp. 417-428.

CADES/Guinée, 2012, «Projet d’appui à la participation citoyenne au renforcement de la gouvernance locale dans les zones minières du littoral (Région Deboké) ", Rapport de synthèse, Conakry, consulté sur internet : http:// www. cades-guinée. org/document. htm, 10 p.

Center for Research on Local Knowledge, 2007, « Décentralisation et gouvernance locale en Afrique : étude comparative sur l'appropriation de la réforme par les communautés rurales au Mali et au Burkina Faso », Rapport de synthèse, Point Sud, Bamako, consulté sur internet: http://www. ascleiden. nl/pdf/cdplocalgovernancenarrativereport. pdf, 19 p.

CGLU et Cities Alliance, 2013, L'environnement institutionnel descollectivités en Afrique, CGLU Afrique, Rabat (Maroc), 120 p.

Decoster D.-P., 2002, Gouvernance locale, développement local et participation citoyenne, ULB, Bruxelles, consulté sur internet : http:// www. ulb. ac. be/igcat/udtl/pdf/gl2002\%20FR. pdf,, 96 p.

DSRP, 2009, " Côte d'Ivoire : Stratégie de réduction de la pauvreté », Rapport du FMI n 09/156 ; 199 P.

Friedberg, E., 1993, Lepouvoir etla règle :dynamiques del'action organisée, Paris, Seuil.

Ghidouche Y.-A. K. et al, 2016, « La participation citoyenne comme approche innovante de co-création de valeur d'une ville : le cas de la ville d’Alger », CIST2016 proceedings, Alger, consulté sur internet : http://www. cist 2016. sciencesconf. org/77325/document, pp. 11-17.

Guiriobé P. J. A., 2015, « Processus d'identification et de conception des projets de développement local dans les collectivités territoriales ivoiriennes : étude de cas des communes d'Alépé, Bouaké et Bonoua », In Revue Africaine d'Anthropologie, Nyansa-Po, n¹8, EDUCI, Abidjan, pp. 32-45.

Koffi B. E., 2010, « La décentralisation, outil de développement local ? », In RGLL, $n^{\circ} 8$, pp 81-100.

Mercier C., 2008, Participation citoyenne et développement des communautés au Québec : enjeux, défis et conditions d'actualisation, ARUC - ISDC, n 8, Montréal, 78 p.

NDI, 2010, Comprendrela gouvernance locale auBurkina Faso : manuel à l'attention des partis politiques et des élus locaux, NDI/USAID, Ouagadougou, $77 \mathrm{p}$.

Thoenig, J.- C., 1996, « Ladécentralisation du pouvoir local », In Annuaire descollectivités locales, Tome 16, pp. 17-31. 
Wiedermann A., et Gafsi H., 2014, La démocratie locale et la participation des citoyens à l'action municipale en Tunisie, GTZ / CFAD, Tunis, 78 p. 